\title{
Work Function of Vicinal Copper Surfaces
}

\author{
P.J. GoDOWsKI* \\ Institute of Experimental Physics, University of Wrocław, pl. M. Borna 9, 50-204 Wrocław, Poland \\ AND J. ONSGAARD \\ Department of Physics and Nanotechnology, Aalborg University, DK-9220 Aalborg East, Denmark
}

(Received February 10, 2012)

Relative work functions of vicinal surfaces to the (100) plane copper surface were determined from the $\Delta \phi$ changes during adsorption of potassium. The initial value of the work function of the different planes was determined from its saturation value under the condition that it corresponds to the potassium overlayer of the same structure and density. It was found that the work function decreases linearly with step density for this step orientation.

DOI: 10.12693/APhysPolA.123.115

PACS: 73.30.+y, 68.35.B-, 68.35.Ct, 68.43.-h

\section{Introduction}

Steps on metal surfaces are an interesting topic for investigation because they can play a role as a prototype of surface defects which are always present in any surface in nature. Stepped surfaces with well-defined step densities are easily prepared with the use of vicinal surfaces, i.e. crystal surfaces whose surface normal is close to those of low-index planes [1-4]. Experimental and theoretical studies have shown the different chemistry of stepped and smooth surfaces [5-8]. The work function of these surfaces decreases with increasing step density and depends on the step orientation. Such a behavior was interpreted as an effect caused by additional dipole moments at the edge atoms.

Various experiments in many cases show the different reactivity of stepped and smooth copper surfaces [9-11]. In this work we present a simple experiment which clearly shows the difference in work function (and as a consequence its electronic structure) among the vicinal plane to the (100) plane of copper. The (100), (119) and (115) surfaces are regularly stepped copper single crystal planes with the same edges but with different width and as a consequence they have a different step density (Fig. 1).

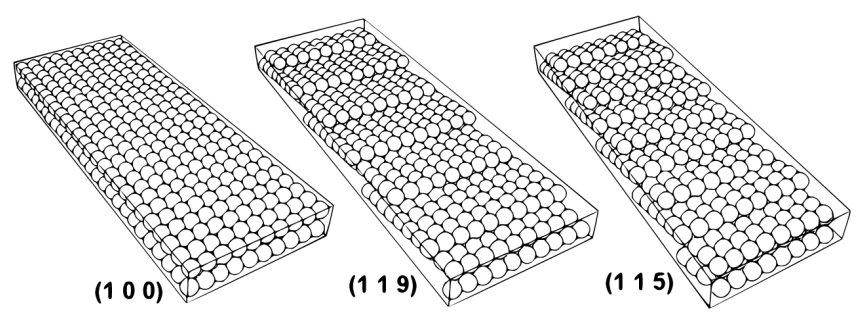

Fig. 1. Model of the (100), (119), and (115) surface of copper.

A correlation between the work function of a surface saturated with an adsorbate and the work function of

\footnotetext{
${ }^{*}$ corresponding author; e-mail: pjg@ifd.uni.wroc.pl
}

the corresponding clean substrate was pointed out in the past [12]. Determination of the work function of the clean surfaces is based on the precise measurements of the work function changes obtained during alkali metal adsorption on the planes at the same experimental conditions (including UHV surroundings, alkali flux, and substrate temperature). From the differences in the saturation level of the $\Delta \phi$, the relative value of the work function of the initial substrate surfaces is derived for all the planes under investigations.

\section{Experimental}

The measurements were performed in the chamber of the beamline SX700 at the ASTRID storage ring, ISA, Aarhus, Denmark. The base pressure during measurements was kept below $1.33 \times 10^{-8} \mathrm{~Pa}$. Full details of the ultrahigh vacuum (UHV) equipment were published elsewhere [13]. The copper sample of size $c a .8 \times 24 \times 1 \mathrm{~mm}^{3}$ was prepared from a single crystal by suitable machining of the metal produced surfaces of the (100), (119) and (115) planes with increasing step density in one direction (Fig. 2). The sample was mounted to the holder on the manipulator enabling cooling to around $110 \mathrm{~K}$ and heating above $1000 \mathrm{~K}$.

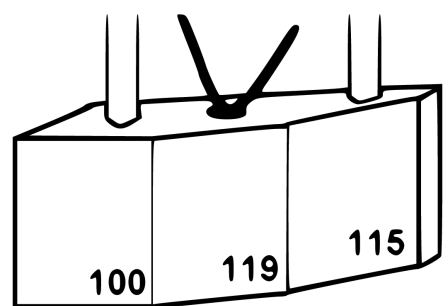

Fig. 2. Schematic drawing of the $\mathrm{Cu}$ sample.

For measurements the sample surface of each plane was positioned (rotation and shift) in such a way that the photon beam impinged the middle of the plane and the plane was perpendicular to the electron energy analyzer. The temperature was controlled by a thermocouple that 
was bent into a hole at the top of the sample (Fig. 2). To remove gaseous impurities, the sample was annealed in UHV. The annealing temperature was kept below $750 \mathrm{~K}$ to avoid outward diffusion of bulk contaminants, mainly sulphur. Then, the sample was cleaned by series of $\mathrm{Ne}^{+}$ ion bombardment (500 eV, $10 \mu \mathrm{A}, 20 \mathrm{~min})$ and annealed (725 K, $5 \mathrm{~min})$. Concerning the (115) orientation, carbon could not be removed completely leaving very small residual signals in the $\mathrm{C} 1 s$ region of the photoemission spectrum. Further ion bombardments could introduce plastic deformation of the surface and a resulting change of the step size and density. The orientation of each plane was checked by low energy electron diffraction and the obtained patterns demonstrated the characteristic features of the stepped surfaces. Although the surface roughness resulting from sputter cleaning is not known, it could be assumed that the density of random steps is small compared to the density of steps on the vicinal planes.

Potassium was evaporated from a SAES GETTERS source placed $c a .50 \mathrm{~mm}$ from the front of the sample. During adsorption the substrate was kept at $123 \mathrm{~K}\left(-150^{\circ} \mathrm{C}\right)$. The flux was evaluated as $5.0 \times 10^{-2}$ $\mathrm{AML} / \mathrm{min}$, where AML denotes adsorbate monolayer with 1 AML corresponding to the most dense potassium overlayer.

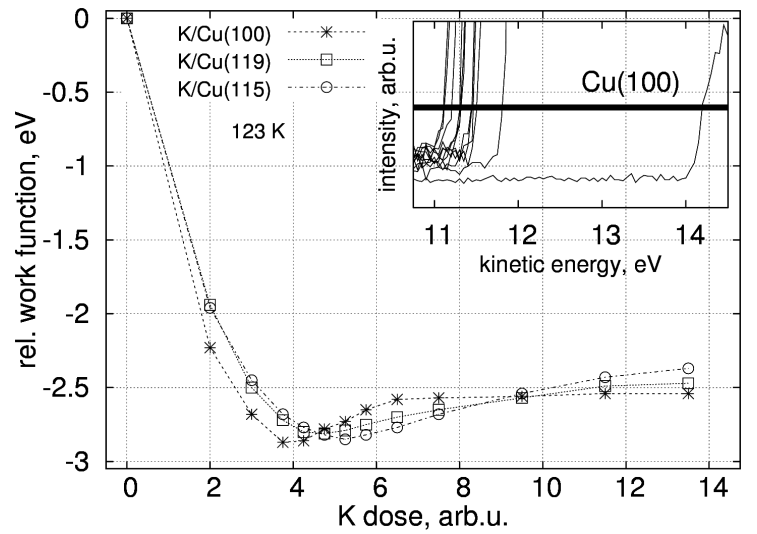

Fig. 3. Work function changes. The inset shows the way of $\Delta \phi$ determination.

Work function changes were measured on the base of the shift of the low energy tail of the secondary electron peak with a sample bias of $-9.00 \mathrm{~V}$ relative to the ground potential [14]. The shift in the secondary peak was measured at a point with a $7.5-10 \%$ (in dependence of the substrate used, but the same for the one series of measurements) intensity of the peak value of the secondary emission peak. The example for the $\mathrm{Cu}(100)$ case is shown in Fig. 3 as the inset; the precision of determination was evaluated as better than $0.01 \mathrm{eV}$.

\section{Results and discussion}

Clean vicinal to $\mathrm{Cu}(100)$ surfaces were investigated by means of two-photon photoelectron spectroscopy by Roth et al. $[15,16]$. A systematic dependence on the step density with the work function difference of $0.050 \mathrm{eV}$ and $0.085 \mathrm{eV}$ for (119) and (115) surface, respectively was published. In the context of above, the work function of the $\mathrm{Cu}(100)$ was given as equal to $4.650(5) \mathrm{eV}$. The details of the cleaning procedure and sample surface preparation during cleaning process were not mentioned. There have been made many experimental and theoretical studies of alkali metal adsorption on copper substrate [17]. In the submonolayer range at room temperature adsorption, the overlayer is influenced by the presence of steps on the substrate and different distinct phases are observed (e.g. $[18,19])$. Potassium deposited at low temperature leads to amorphous, but homogeneous films whereas growth at room temperature results in a motion of disjoined crystalline islands. Due to big difference between lattice constants of potassium (bcc, $532.8 \mathrm{pm}$ ) and copper (fcc, $361.49 \mathrm{pm}$ ), in principle, the full monolayer structure of alkali does not depend on the substrate orientation. Low temperature (123 K) adsorption of potassium on the $\mathrm{Cu}(100)$ leads to the StranskiKrastanov deposition mode, i.e. after completion of 1-3 layers, the growth is $3 \mathrm{D}$ islands [20]. It is expected that the complete monolayer of $\mathrm{K}$ on the (100), (119) and (115) copper substrates has the structure of the most dense adsorbate plane, i.e. the K(110) surface with a density of $4.98 \times 10^{18}$ at. $/ \mathrm{m}^{2}$.

The work function changes obtained during potassium adsorption at $123 \mathrm{~K}$ on the "triple-plane" copper surface are reproduced in Fig. 3. The inset shows the plots of the low energy tail of photoelectron spectra $(h \nu=300 \mathrm{eV})$ referring to the (100) plane which were used in $\Delta \phi$ determination. In each case, the work function changes follow the well known routine. The beginning of $\Delta \phi$ could be approximated by a linear dependence with coverage. From the slope of the first part of the graph, the initial dipole moment of the potassium ions acquires the value from the 9.8-8.0 D interval as determined from the Helmholtz equation [21]. Then follows the more or less deeper minimum and at the end the saturation. The extent of the minimum corresponding to the stepped surface is smaller and it could be caused by the presence of residual carbon contamination. The most exact and reliable value of $\Delta \phi$ is that at saturation. Although it corresponds to the same, most dense, potassium monolayer, the saturation value varies for the different planes. It is induced by differences in the work function of the clean surfaces under investigation. The are collected in Table. The uncertainty was evaluated from the scatter of two last points.

According to the presented framework, the differences in the saturation value of the work function could be treated as the differences between the work function of the initial surfaces. Figure 4 shows the relation between the relative value of the work function with the step density. It is seen that the work function decreases linearly with increasing step density, i.e. $\phi(100)>\phi(119)>$ $\phi(115)$; all in the range of $0.15 \mathrm{eV}$. 
TABLE

Step densities of copper surfaces, $n_{\mathrm{s}}$, and the mean value of the work function at saturation $\overline{\Delta \phi}$.

\begin{tabular}{c|c|c|c}
\hline \hline Plane & Nomenclature & $n_{\mathrm{s}}\left[\mathrm{m}^{-1}\right]$ & $\overline{\Delta \phi}[\mathrm{eV}]$ \\
\hline$(100)$ & $\infty(100) \times(111)$ & 0 & $-2.54(2)$ \\
$(119)$ & $5(100) \times(111)$ & $8.59 \times 10^{8}$ & $-2.48(2)$ \\
$(115)$ & $3(100) \times(111)$ & $1.51 \times 10^{9}$ & $-2.40(2)$
\end{tabular}

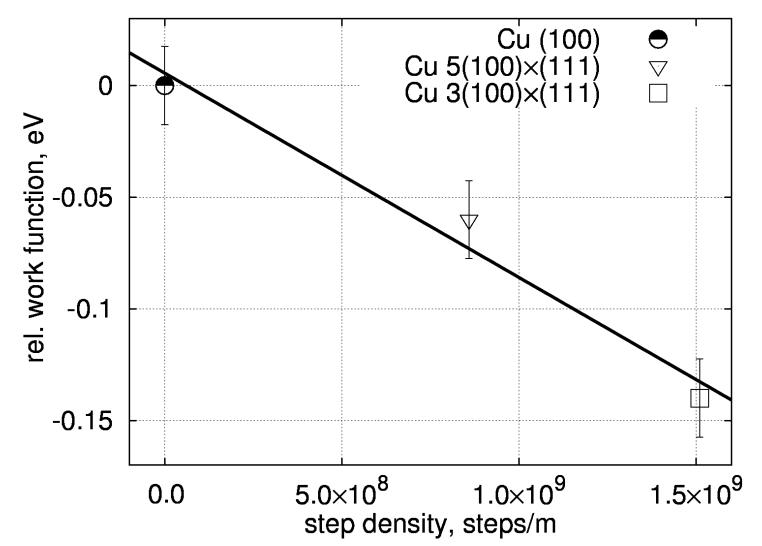

Fig. 4. Relative work function of vicinals of $\mathrm{Cu}(100)$.

The effect of atomic scale surface roughness is interpreted in terms of the smoothing of the electronic charge density parallel to the surface [5]. This smoothing leads to a more positive surface potential and a lowering of the work function.

\section{Acknowledgments}

The research leading to these results acknowledged funding from European Community's Seventh Framework Programme (FP7/2007-2013) under grant agreement no. 226716.

\section{References}

[1] H. Ishida, A. Liebsch, Phys. Rev. B 46, 7153 (1992).

[2] Z.-J. Tian, T.S. Rahman, Phys. Rev. B 47, 9751 (1993)
[3] D. Spišák, Surf. Sci. 489, 151 (2001)

[4] P. Hecquet, Surf. Sci. 604, 834 (2010)

[5] B. Krahl-Urban, H. Wagner, R. Butz, Surf. Sci. 93, 423 (1980)

[6] J.F. Jia, K. Inoue, Y. Hasegawa, W.S. Yang, T. Sakurai, Phys. Rev. B 58, 1193 (1998)

[7] Z.L. Mišković, S.G. Davison, F.O. Goodman, Phys. Rev. B 60, 2025 (1999).

[8] I. Merrick, J.E. Inglesfield, G.A. Attard, Phys. Rev. B 71, 085407 (2005)

[9] L. Bech, J. Onsgaard, S.V. Hoffmann, P.J. Godowski, Surf. Sci. 482, 243 (2001)

[10] J. Onsgaard, L. Bech, C. Svensgaard, P.J. Godowski, S.V. Hoffmann, Prog. Surf. Sci. 67, 205 (2001)

[11] J. Onsgaard, S.V. Hoffmann, P. Møller, P.J. Godowski, J.B. Wagner, G. Paolucci, A. Baraldi, G. Comelli, A. Groso, Chem. Phys. Chem. 4, 466 (2003).

[12] X.Q.D. Li, R. Vanselow, Surf. Sci. 236, L369 (1990)

[13] J. Onsgaard, L. Thomsen, S.V. Hoffmann, P.J. Godowski, Vacuum 81, 25 (2006)

[14] M.G. Helander, M.T. Greiner, Z.B. Wang, Z.H. Lu, Appl. Surf. Sci. 256, 2602 (2010).

[15] M. Roth, M. Pickel, J. Wang, M. Weinelt, Th. Fauster, Appl. Phys. B 74, 661 (2002)

[16] M. Roth, T. Fauster, M. Weinelt, Appl. Phys. A 88, $497(2007)$

[17] R.D. Diehl, R. McGrath, Surf. Sci. Rep. 23, 43 (1996).

[18] H.L. Meyerheim, J. Wever, V. Jahns, W. Moritz, P.J. Eng, I.K. Robinson, Surf. Sci. 304, 267 (1994)

[19] M.-S. Chen, S. Mizuno, H. Tochihara, Surf. Sci. 601, $5162(2007)$

[20] P.J. Godowski, A. Groso, S.V. Hoffmann, J. Onsgaard, Acta Phys. Pol. A 117, 928 (2010).

[21] E.V. Albano, H.O. Martin, Phys. Rev. B 35, 7820 (1987) 